\title{
Chondrosarcoma in the mental foramen region of the mandible: A case report
}

\author{
HONGWEI LIU ${ }^{1}$, XIUHONG CHEN ${ }^{1}$, TENG WAN ${ }^{2}$ and REN LI ${ }^{3}$ \\ ${ }^{1}$ Department of Oral and Maxillofacial Surgery, Jining First People's Hospital, Jining, Shandong 272100; \\ ${ }^{2}$ Department of Oral and Cranio-Maxillofacial Science, Ninth People's Hospital, \\ Shanghai Jiaotong University School of Medicine, Shanghai 200011; ${ }^{3}$ Department of Pathology, \\ Jining First People's Hospital, Jining, Shandong 272100, P.R. China
}

Received December 28, 2014; Accepted January 27, 2016

DOI: $10.3892 / \mathrm{ol} .2016 .4863$

\begin{abstract}
Chondrosarcoma is a malignant tumor that is characterized by the formation of cartilage by tumor cells. The tumor is commonly observed in regions where cartilage is already present. In total, 5-12\% of chondrosarcomas occur in the head and neck region. However, the occurrence of chondrosarcoma in the mental foramen region is extremely rare. The aim of the present study was to report a case of chondrosarcoma in the mental foramen region. An 18-year-old man presented with a 3-month history of a painless mass over the labial aspect of the right mandible, which was associated with the patient feeling unwell and the loosening of the lower anterior teeth. The patient underwent segmental mandibulectomy and the final pathological diagnosis was chondrosarcoma. Following mandibulectomy, the patient continued to receive routine follow-up. In addition, the present study reviewed the literature concerning chondrosarcomas in the head and neck region.
\end{abstract}

\section{Introduction}

Chondrosarcoma is a malignant tumor characterized by the formation of cartilage by tumor cells (1). Chondrosarcomas have been widely reported in the literature and may arise in any region where cartilage is present (2). In total, 5-12\% of chondrosarcomas occur in the head and neck region, accounting for 5-10\% of all bone neoplasms occurring in this location; the larynx and maxillo-nasal region are the most common sites for chondrosarcomas to originate (3). Chondrosarcomas located in the mandible region are rare and mostly occur in the mandibular symphyseal region (4-6). The majority of chondrosarcomas exhibit an indolent growth pattern, and more aggressive, high-grade tumors have been

Correspondence to: Miss. Xiuhong Chen, Department of Oral and Maxillofacial Surgery, Jining First People's Hospital, 6 Jiankang Road, Jining, Shandong 272100, P.R. China

E-mail: liuqufu2004@126.com

Key words: chondrosarcoma, mandible, mental foramen region, immunohistochemistry previously observed (7). Forming a pre-operative diagnosis for chondrosarcomas is challenging due to the atypical symptoms and imaging manifestations; therefore, diagnosis primarily depends on the post-operative pathological examination. To date, the clinical course, histogenesis, cytogenetics and prognosis for chondrosarcomas remain largely unknown (8). The treatment for the tumor depends on the outcome of surgical resection, and the prognosis for patients with chondrosarcomas is good when the tumors are completely resected (9). The occurrence of chondrosarcoma in the mental foramen region of the mandible is an exceptional event. The present study describes the case of a patient with chondrosarcoma in the right mental foramen region of the mandible. Written informed consent was obtained from the patient for the publication of the present study.

\section{Case report}

An 18-year-old man was referred to Jining First People's Hospital (Jining, China) in December 2009 with swelling over the labial aspect of the right mandible that had occured for $\sim 3$ months. The swelling had increased gradually in size during that period. The patient did not have a history of trauma or pain, but reported that the anterior mandible felt swollen and that there was a slight loosening of the lower anterior teeth.

Extraoral examination demonstrated no sign of facial swelling or asymmetry. No lymph node involvement was observed, and all the cranial nerves were uninjured, since there was no facial palsy observed. Intraoral examination revealed a swelling, $\sim 1.5 \times 2.0 \times 2.0 \mathrm{~cm}$ in size, at the sulcus vestibularis of the lower right canine and lateral incisor. The hard, painless mass was fixed to the mandible and covered with normal mucosa. No swelling was observed at the lingual aspect. The mandible swelling had caused displacement of the crown of the lower right canine and lateral incisor; however, the patient experienced no numbness or limitation in mouth opening.

Panoramic radiographs revealed a radiolucent lesion with irregular bone diffusion, which displaced the roots of the lower right canine and lateral incisor. Next, 64-multidetector-row computed tomography (CT) (Somatom Definition AS; Siemens Medical Solutions, Forchheim, Germany) demonstrated bone absorption around the roots of the lower central 


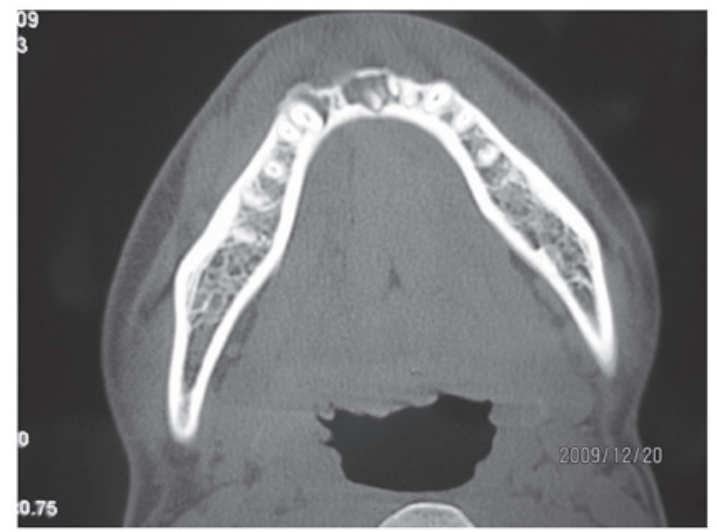

Figure 1. Computed tomography scan revealing bone destruction surrounding the roots of the lower central incisors and right lateral incisor, and a widening of the periodontal ligament space.

and lateral incisors, and a widening of the parodontium (Fig. 1). Three-dimensional imaging (Somatom Definition AS; Siemens Medical Solutions) revealed that there was destruction of the bone at the right mental foramen region of the mandible (Fig. 2).

The patient was advised to undergo surgery, and the tumor was resected by segmental mandibulectomy between the lower right first molar and the lower left first premolar. The defect in the mandible was reconstructed with a free vascularized fibula flap.

Resected tissue specimens were formalin-fixed, paraffin-embedded, cut into $5-\mu \mathrm{m}$ sections and stained with hematoxylin and eosin (Sigma-Aldrich, St. Louis, MO, USA). Post-operative histopathological examination of the mandibular lesion demonstrated that the lesion was a neoplasm composed of lobular cartilage cell clumps, which had invaded the surrounding tissue. Under low magnification (x40), the tumor cells were lobulated with rich chondromyxoid stroma. The tumor cells were basophilic and infiltrated the surrounding normal bone tissue. Under high magnification (x200), the tumor cells were abundant and of various sizes. The nuclei of the cells were enlarged and were heavily stained, with moderate heterotypical alterations compared with normal cells. Binuclear cells were rarely observed (Fig. 3). Cobas ${ }^{\circledR}$ CORE II Automated Immunity System (Roche Diagnostics GmbH, Mannheim, Germany) was used to quantitatively detect the levels of the $\mathrm{S}-100$ protein. The slides were blocked with $10 \%$ goat serum (ZSGB-BIO, Beijing, China) for $30 \mathrm{~min}$, then incubated with mouse anti-human monoclonal S-100 antibody (cat. no. Kit-0007; dilution, 1:100; Fuzhou Maixin Biotechnology Co., Ltd., Fuzhou, China). Immunohistochemistry demonstrated that $80 \%$ of the tumor cells exhibited S-100 protein (Fig. 4). A diagnosis of grade II chondrosarcoma was provided as a result (10).

The post-operative course was uneventful and the patient was not referred for radiation therapy, since all margins of the resected region were tumor-free. Post-treatment follow-up evaluations every 6 months indicated no evidence of recurrence 3 years following surgery, as observed by CT. There was no evidence of recurrence 3 years after surgery, and the patient continues to undergo routine follow-up examinations.

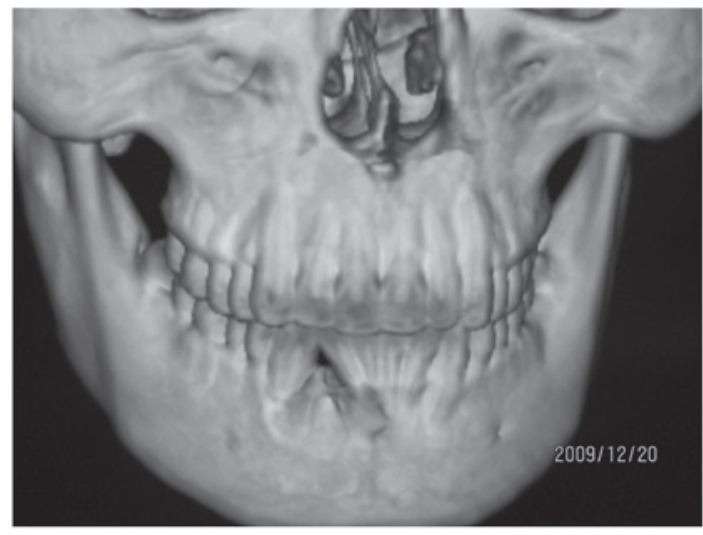

Figure 2. Three-dimensional image revealing bone resorption at the mental foramen region of the mandible.

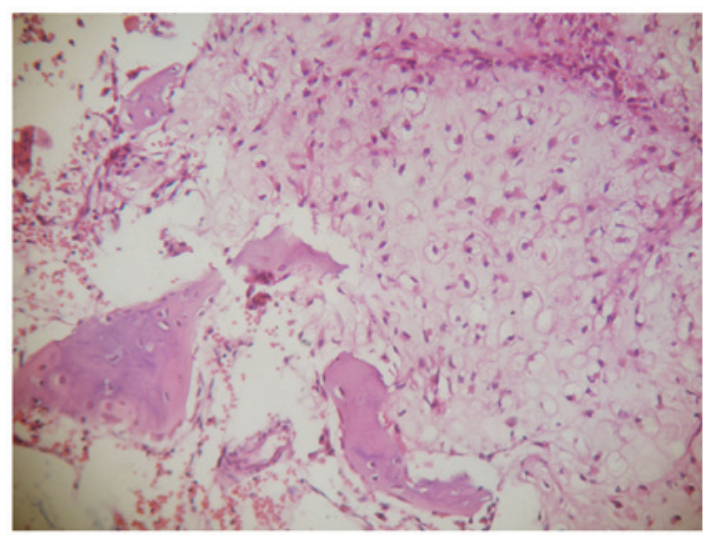

Figure 3. High-magnification photomicrograph demonstrating mitosis in a few cells (hematoxylin and eosin staining; magnification, x200).

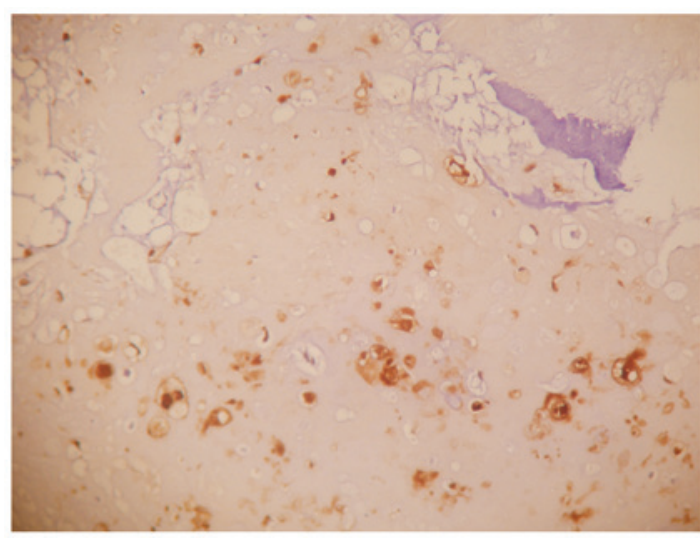

Figure 4. Positive expression of S-100 protein in the nucleus and cytoplasm of the tumor cells (magnification, $\mathrm{x} 200$ ).

\section{Discussion}

Chondrosarcoma accounts for $\sim 11 \%$ of all primary malignant bone tumors. The tumor is commonly observed in the pelvic bones, the proximal femur, the proximal humerus, the distal femur and the ribs; $5-12 \%$ of chondrosarcomas occur in the head and neck region (11). The most common sites for chondrosarcoma in the head and neck region have been reported as the jaw bones, 
paranasal sinuses, nasal cavity, maxilla and vertebrae $(3,12,13)$. The occurrence of chondrosarcoma in the mandible is rare. The molar region and, in decreasing order, the symphysis and the coronoid process may be involved, but occurrence in the mental foramen region is extremely uncommon $(12,14)$. Chondrosarcoma in the mandible is characterized by the presence of a swelling mass with or without pain, loosening and displacement of involved teeth, and widening of the parodontium (4). Clinical features, including loss of nerve sensation and dysesthesia, are used to distinguish a malignant neoplasm from osteomyelitis.

Radiographically, chondrosarcomas exhibit a range of features consistent with their malignant nature. In general, the margins of the lesion are ill-defined, with a holey, lytic appearance. Radiographical findings provide a certain amount of evidence for the diagnosis of chondrosarcoma, such as loosening of the involved teeth and widening of the periodontal ligament space (4).

The final diagnosis relies on histological and immunohistochemical examination of the lesion. Low magnification microscopy reveals a spectrum of findings, which are similar to those of chondrosarcomas located in other regions of the body. The lesions appear lobulated and infiltrate the surrounding normal bone tissue. Under high magnification microscopy, the tumor cells exhibit a hyaline cartilaginous proliferation, with a sarcomatous stroma containing stellate, spindle-shaped or round cells. Cell nuclei are enlarged, heavily stained and have moderate heterotypical alterations. The presence of binuclear cells is rare. Immunohistochemical analysis demonstrates that tumor cells express the S-100 protein. The study by Evans et al (10) classified chondrosarcoma into three grades (I, II and III), based on the frequency of mitosis, cellularity and the dimensions of the nuclei. The present case was classified as grade II chondrosarcoma. It may be difficult to distinguish well-differentiated chondrosarcoma (grade I or II) from a chondroma as chondrosarcomas exhibit various histological patterns, which range from undifferentiated neoplasms to benign chondroid tumors.

Wide surgical resection is known to be the most effective treatment for chondrosarcoma (1). For these tumors, local recurrence is more common than distant metastasis (15), and there is a relatively good association between tumor grade and prognosis. Tumor grade and resectability are the most important prognostic factors for head and neck chondrosarcomas (9). These factors make the early diagnosis and complete resection of the tumor extremely important for the prognosis of the patient (16). Radiotherapy for chondrosarcoma is generally accepted as an adjuvant therapy in cases of residual disease rather than as the initial treatment $(17,18)$.

Overall, chondrosarcoma is a malignant tumor with a relatively low rate of lymph node involvement or distant metastasis. Therefore, an early diagnosis and complete resection are extremely important for a good prognosis, and elective neck dissection is not usually required. Radiotherapy is only recommended for adjuvant purposes when the margin is positive or the tumor is unresectable. In the present case, the patient underwent successfully underwent a resection only, and there was no evidence of disease at 3 years post-surgery.

\section{References}

1. Koch BB, Karnell LH, Hoffman HT, Apostolakis LW, Robinson RA, Zhen W and Menck HR: National cancer database report on chondrosarcoma of the head and neck. Head Neck 22: 408-425, 2000

2. Garrington GE and Collett WK: Chondrosarcoma. I. A selected literature review. J Oral Pathol 17: 1-11, 1988.

3. Burkey BB, Hoffman HT, Baker SR, Thornton AF and McClatchey KD: Chondrosarcoma of the head and neck. Laryngoscope 100: 1301-1305, 1990.

4. Saini R, Abd Razak NH, Ab Rahman S and Samsudin AR: Chondrosarcoma of the mandible: A case report. J Can Dent Assoc 73: 175-178, 2007.

5. Izadi K, Lazow SK, Solomon MP and Berger JR: Chondrosarcoma of the anterior mandible. A case report. NY State Dent J 66: 32-34, 2000.

6. Ormiston IW, Piette E, Tideman H and Wu PC: Chondrosarcoma of the mandible presenting as periodontal lesions: Report of 2 cases. J Craniomaxillofac Surg 22: 231-235, 1994.

7. Riedel RF, Larrier N, Dodd L, Kirsch D, Martinez S and Brigman BE: The clinical management of chondrosarcoma. Curr Treat Options Oncol 10: 94-106, 2009.

8. Sammartino G, Marenzi G, Howard CM, Minimo C, Trosino O, Califano L and Claudio PP: Chondrosarcoma of the jaw: A closer look at its management. J Oral Maxillofac Surg 66: 2349-2355, 2008.

9. Lee SY, Lim YC, Song MH, Seok JY, Lee WS and Choi EC: Chondrosarcoma of the head and neck. Yonsei Med J 46: 228-232, 2005.

10. Evans HL, Ayala AG and Romsdahl MM: Prognostic factors in chondrosarcoma of bone: A clinicopathologic analysis with emphasis on histologic grading. Cancer 40: 818-831, 1977.

11. Schajowicz F, Ackerman LV and Sissons HA (eds): Histological Typing of Bone Tumors. International Histological Classification of Tumors. 2nd edition. World Health Organization, Geneva, 1972.

12. Weiss WW Jr and Bennett JA: Chondrosarcoma: A rare tumor of the jaws. J Oral Maxillofac Surg 44: 73-79, 1986.

13. Ruark DS, Schlehaider UK and Shah JP: Chondrosarcomas of the head and neck. World J Surg 16: 1010-1016, 1992.

14. Hackney FL, Aragon SB, Aufdemorte TB, Holt GR and Van Sickels JE: Chondrosarcoma of the jaws: Clinical findings, histopathology and treatment. Oral Sur Oral Med Oral Pathol 71: 139-143, 1991.

15. Sesenna E, Tullio A and Ferrari S: Chondrosarcoma of the temporomandibular joint: A case report and review of the literature. J Oral Maxillofac Surg 55: 1348-1352, 1997.

16. Tullio G and D'Errico P: Chondrosarcoma of the mandible. Clinical and histological considerations. Ann Stomatol (Roma) 23: 191-206, 1974 (In Italian).

17. Harwood AR, Krajbich JI and Fornasier VL: Radiotherapy of chondrosarcoma of bone. Cancer 45: 2769-2777, 1980.

18. McNaney D, Lindberg RD, Ayala AG, Barkley HT Jr and Hussey DH: Fifteen year radiotherapy experience with chondrosarcoma of bone. Int J Radiat Oncol Biol Phys 8: 187-190, 1982. 